\title{
Les sentiments religieux et mystiques \\ Chez Alphonse de Lamartine
}

par

\section{Dr. Siham Abd-el Fattah Mohamed Gabr}

Professeur adjoint de la littérature française - Faculté des lettres

Université de ZAGAZIG

\section{Siham_gabr83@zu.edu.eg}

\section{Resumeè:}

Lamartine, au sens le plus large du mot, joue un grand rôle à cet effet ; mais il faut, mettre en relief l'influence du milieu environnant et spécialement celle de sa famille.

La première influence qu'il a subie est sans doute celle de sa mère éprise et possédée du Sacre. Avec quelle ferveur, Lamartine évoque ces scènes pathétiques quand sa mère lit ce petit volume de psaumes dont les pages " feuilletées à toutes les circonstances de sa vie. C'est ainsi que Lamartine, réceptif à toutes les réminiscences de sa vie, le sera particulièrement pour sa jeunesse. Le grand lot de ses souvenirs sera réserve à sa mère dont les leçons sont saintes.

En quelques mots, on peut dire que Lamartine, était "un poète maître de son discours, un poète délicat, plus sensible que sensuel ,grâce aux différents aspects du mysticisme religieux, déjà analysés, il exprime en beaux vers, des sentiments purs et pénétrant, inondant et forts .

Le mysticisme religieux fait de lui un poète chaste, sans effort et sans gaucherie puisqu' il le détail d'une distinction innée qui le menait, sans qu'il y songeât peut-être, à choisir

سهام عبد القتاح محمد جبر (Les sentiments religieux et mystiques Chez Alphonse de Lamartine) 
parmi les émotions, les plus nobles et les plus sincères comme l'on vient de voir.

\section{Introduction:}

Alphonse de Lamartine, né à Mâcon le 21/10/1790 et mort à Paris le 28/02/1869 est un poète, romancier, dramaturge français.

Le sentiment religieux fut intense Chez Lamartine dès son enfance. On lui avait appris à reconnaître Dieu dans les moindres événements de la vie, et jusqu' à sa mort, il gardera la vivacité et l'ardeur de cet amour pour Dieu. Ce fut dans les premières années de sa vie trop un sentiment et rien que cela. Cependant, à tout propos, dans sa vie privée, dans sa vie politique, dons ses œuvres littéraires, il exhalera naturellement et spontanément un sentiment de mysticisme religieux.

En effet, son âme mystique traduit ses élans dont la sincérité est toujours émouvante. Son éducation, sa noblesse d'esprit ont fait de lui un être désintéressé et généreux. Tout ce qui est beau, tout ce qui est délicat, tout ce qui est grand le séduit et l'attire.

\section{Origine de ce mysticisme religieux :}

Il va sans dire que la formation littéraire et artistique de Lamartine, au sens le plus large du mot, joue un grand rôle à cet effet; mais il faut, mettre en relief l'influence du milieu environnant et spécialement celle de sa famille.

La première influence qu'il a subie est sans doute celle de sa mère éprise et possédée du Sacre. Avec quelle ferveur, Lamartine évoque ces scènes pathétiques quand sa mère lit ce petit volume de psaumes dont les pages " feuilletées à toutes les circonstances de sa vie, portaient l'empreinte de ses doigts et quelques traces de ses larmes." 
C'est ainsi que Lamartine, réceptif à toutes les réminiscences de sa vie, le sera particulièrement pour sa jeunesse. Le grand lot de ses souvenirs sera réserve à sa mère dont les leçons sont saintes:

"Mon oreille s'ouvrait à tes accents si doux que du monde et du ciel l'obscure intelligence à travers ton sourire éclairait mon enfance que tes saintes leçons façonnait ma raison " 2

C'est surtout la mère qui inspirera au Poète les plus profonds préceptes de son mysticisme.

Il avoue dans les Confidences que, sans elle, il n'aurait rien su "épeler de la création " qui avait sous les yeux " 3

Cette mère pieuse et ses leçons resteront gravées dans le cœur de Lamartine. Il suffit pour une âme sensible de visiter les lieux saints pour que se ravivent les flammes de cette éternelle piété :

"Cette foi qui m'attend au bord de mon tombeau

Hélas! il m'en souvient, plana sur mon berceau

De la terre promise immortel héritage

Les pères à leurs fils l'ont transmis d'âge en âge

Notre esprit la reçoit à son premier réveil

Comme les dons d'en haut, la vie et le soleil

Comme le lait de l'âme en ouvrant la paupière

Elle a coulé pour nous des lèvres d'une mère

Elle a pénétré l'homme en sa tendre saison "14

C'est ainsi que le poète a été très marqué par cette éducation religieuse hautement qualifiée. Il ne cessera de transmettre son heureuse expérience aux autres :

" La piété de nos premiers jours rentre dans notre âme pour ainsi dire par les sens avec la mémoire de notre berceau, de notre prière du premier foyer

سهام عبد الفتاح محمد جبر (Les sentiments religieux et mystiques Chez Alphonse de Lamartine) 
du premier temps où l'on a appris à épeler le nom que nos parents donnaient à Dieu." ${ }^{5}$

L'influence maternelle qui implique les meilleurs préceptes d'un sentiment religieux portera ses fruits . Le vieillard dans $\underline{\mathbf{l a}}$ Chute d'un Ange, sera témoin de cette reconnaissance touchante :

“ ma mère m'enseignait à soulager mes frères, à panser leur blessure, à porter leur fardeau, à donner à leur soif l'huile ou la goutte d'eau pour ne pas augmenter ma misérable Caste. Nos prières montaient de ses lèvres de femme." ${ }^{6}$

Quant au père, il suffit de signaler que la prière qu'il faisait restera gravée dans la mémoire de Lamartine. La voix de son père lui rappelait "l'accent religieux des psalmodies du prêtre le dimanche dans l'église de Milly " , "tout cela -dit-il suscitait vivement mon attention, ma curiosité , mon émotion même ." 7

En réalité, Lamartine ne cesse de parler de la vertu que lui enseignait son père ni du sentiment religieux qu'il lui a inculque.${ }^{8} \mathrm{Il}$ va même jusqu' à dire que le père lui enseignait la foi par la Reconnaissance. ${ }^{9}$

A joutons aussi que Lamartine a été influence par les pères qui lui enseignaient à l'école, surtout par les jésuites de Belley, Le Père Varlet, le cure de Bussières. Lamennais a eu sur sa formation mystique et religieuse une influence particulière. La lecture de L'Essai sur l'Indifférence avait frappé son âme, comme il le signale dans les Méditations:

"L'Essai sur l'Indifférence m'avait frappé comme une page de J.J.Rousseau retrouvée dans le XIXe siècle. Je m'attachais aux arguments qui me paraissaient faibles, mais l'argumentation me ravissait... j'avais besoin d'épancher mon

سهام عبد القتاح محمد جبر (Les sentiments religieux et mystiques Chez Alphonse de Lamartine) 
admiration; je ne pouvais le faire qu'en m'élevant au sujet le plus haut de la pensée humaine : Dieu ." 10

En réalité , cette influence approfondira le sens religieux et mystique chez lui ; c'est surtout dans les Méditations que se manifestent les empreintes de cette recrudescence mystique . Son caractère pondéré et réfléchi marque une personnalité prestigieuse. Dans une méditation intitulée Dieu, dédiée à l'abbé Lamennais, le poète reconnait la juste place du Créateur dans l'Univers depuis les temps les plus avances de la Création . La générosité révélatrice de la Providence y est nette et claire . Le pardon est la devise de la loi divine. Si Les êtres humains se montrent ingrats et oublieux des messages divins, le Créateur leur offrira une nouvelle chance, en renouvelant son rappel par l'intermédiaire des anges.Tel est le fond de la méditation "Dieu" rédigée en mai : 1819

"L 'homme dans le passe ne remontait qu' à toi

Longtemps comme un enfant, ta voix daignera

M'instruire et par la main longtemps tu voulus le conduire Que de fois dans la gloire à lui tu t'es montre Aux vallons de Sennar, aux chênes de Membre Dans le buisson d'Horeb ou sur l'auguste cime Ou Moise aux Hébreux dictait sa loi suprême Ces enfants de Jacob, premiers nés des humains Reçurent quarante ans la manne de tes mains Tu frappais leur esprit par tes vivants oracles Tu parlais à leurs yeux par la voix des miracles Et lorsqu' ils t'oublieraient les anges descendus Rappelaient ta mémoire à leurs cours éperdus "11

En effet, c'est grâce à Lamennais que le sentiment du mysticisme religieux a été renforcé chez Lamartine. Aussi, ne doit-on pas négliger l'influence de la Bible dont l'imagination du poète est imprégnée ; il suffit de voir un simple paysage

سهام عبد الفتاح محمد جبر (Les sentiments religieux et mystiques Chez Alphonse de Lamartine) 
pour que s'éveille dans sa tête une image biblique, comme lorsqu'il dit:

"Les saints . les poètes, les sages

Ecouteront dans nos l'feuillages

Des' bruits pareils aux grandes eaux." 12

D'autre part, il faut signaler que Lamartine a beaucoup admiré Fénelon à cause de son amour désintéresse de Dieu, cet amour ne demande à l'adoration d'autre récompense que l'adoration.

Il y a aussi l'influence du poète anglais du XVIIe siècle "Milton"13. En effet, le Paradis perdu de Milton a attiré l'attention et 1 'admiration de Lamartine qui s'en souviendra dans la Chute d'un Ange lorsqu'il dit:

"Que du Tigre qui rampe au passereau vole

Chacun se réjouisse à l'humaine parole

Et les loups dévorants sortiront des forets

Et la colère et l'agneau se coucheront auprès

Et de tout ce qui vit la sagesse infinie

Rétablira d'Eden la première harmonie ." 14

Ce qui est vraiment bizarre, c'est que Lamartine luimême reconnaît, dans ses Cours Familiers de littérature que son mysticisme religieux a subi l'influence de la littérature indienne; il dit:

"L'Inde m'avait révélé une plus large charité de l'esprit humain, la charité envers la nature entière." 15

En réalité, Lamartine n'oubliera pas les leçons des Brahmanes, théologiens et philosophes de l'Inde:

'Rêves pour rêves, nous aimerions mieux rêver avec les Brahmanes, ces théologiens philosophes de l'Inde primitive, ces précurseurs de la philosophie chrétienne." 16

سهام عبد الفتاح محمد جبر (Les sentiments religieux et mystiques Chez Alphonse de Lamartine) 
En effet, la pensée des Brahmanes indiens acquiert une importance particulière puisqu'elle est basée essentiellement sur le mysticisme religieux . En .lisant les textes de la littérature de l'hindouisme, Lamartine découvre cette espèce de charité basée sur un mysticisme religieux quelle que soit cette religion

:-"Cette charité , dit-il, a tout prix qui est le caractère de ces poésies sacrées de l'Inde et qui est Le pressentiment d'une autre charité, est bien supérieure à la justice . "17

Si nous ajoutons à cas différentes influences la nature même de l'âme de Lamartine, on comprendra, sans beaucoup de peine, le fond de son mysticisme religieux. mais quels sont les divers aspects de cette tendance et comment les découvre-ton dans ses œuvres?

En effet, on doit signaler, avant tout que, chez Lamartine, rien ne se sent l' effort car, pensons-nous, son œuvre n'est que la respiration naturelle de son âme . Tout est spontané ; la poésie pour lui est ce qu'est la prière; une Méditation pour lui n'est qu'un soupir d'une Harmonie n'est que l'exclamation naturelle et incompressible d'une âme éprise d'infini . C'est dans ce cadre qu'on mettra en relief les différentes manifestations de ce mysticisme religieux de Lamartine.

\section{Les manifestations de se mysticisme religieux :}

\section{La lumière :}

Le premier trait happée des sens, cet accuse par la perception visuelle. Lamartine dans ses transports mystiques se confond avec l'éternité. Il ne voit que la lumière qui l'absorbe, l'emporte dans son élan pendant ces moments d'amalgamation avec l'irradiation du Cosmos, représentant le paroxysme de sa

سهام عبد القناح محمد جبر (Les sentiments religieux et mystiques Chez Alphonse de Lamartine) 
béatitude spirituelle, et qui dit spirituel peut dire physique si l'on prend en considération l'influence sensationnelle de l'esprit sur le corps.

Lamartine se dissout dans ces éclairs de lumière, y disparaît; dans ces moments d'extase et de ravissement spirituel. Ces transes ne sont pas sans une grande félicité qui pétrit le physique dans le spirituel, en le niant et ainsi elles procurent à Lamartine des sensations délicieuses:

"Ce sont de ces moments d'ineffables délices dont Dieu ne laisse pas épuiser les calices des éclairs de lumière et de félicité qui confondent la vie avec l'éternité." 18

Relevons bien "délices" et félicite" deux sensations parfaitement expressives de l'adhésion du matériel au spirituel dans ces moments d'enthousiasme culminant et de dissolution totale dans Le Cosmos.. Quand son âme s'envole dans ces heureux moments d'ataraxie, de sérénité et d'extase, toutes les notions des ténèbres se dissipent. Un monde permanent de lueur et de lumière apparaît, sorte d' irradiation continue du jour. Lamartine souhaite. La pérennité d'une telle situation et ne se contente pas de ces phénomènes mystiques. Cela explique l'ardeur et la ferveur qui l'envahisse pour s'émanciper définitive ment de son enveloppe charnelle:

"Quand pourrez-vous mes yeux, quand pourrez-vous, hélas! saluer les splendeurs divines du jour qui ne s'éteindra pas?

(...) Dieu du jour. Dieu des nuits! Dieu de toutes les heurs laisse-moi m' envoler sur les feux du soleil! ou va vers l'accident ce nuage vermeil? Il va voiler le seuil de tes saintes demeures, ou l'œil ne connaît plus la nuit ni le sommeil." 19 
Dès que la perception de la clarté est réalisée, le poète enthousiasmé par son transport, se transfère tout à fait dans l'autre monde qu'il cherche incessamment, afin d'abandonner une vie dépourvue de tout attrait significatif et d'où n'émane qu'une fadeur fastidieuse, Alors, son cœur s'anime par les trouvailles longtemps recherchées et une indescriptible ferveur gagne son être et flamboie ses entrailles par une tornade de sentiments qui ne participent pas de ce bas monde. Dans la mêlée de ses acquisitions grisantes, ébloui par la lumière, il se pose tant de questions, tellement l'irréalisable est devenu concret a sa perception sensorielle. Toutefois, il devine aisément que cette lumière n'est autre qu'une irradiation des mânes flottant en pleine liberté dans leur monde joyeux:

"Mon cour à ta clarté s'enflammé

je sens des transport inconnus

je songe à ceux qui ne sont plus

douce lumière, es-tu leur âme." 20

Dans son transport mystique le poète atteint cette phase transcendante ou n'existe que la lumière qui l'unira aux mânes paisibles :

"enveloppé de leur image

je crois me sentir plus près d'eux!" 21

Cet état de dégagement absolu de tout ce qui est matériel se conjuguera foncièrement avec toute source de lumière; éclair, étincelle, ... qui lui permettront de rejoindre le monde de ceux qui l'ont quitté.

Cela représente la première phase du transport mystique qui sera étroitement liée à la seconde: celle de la "transparence".

\section{- La Transparence.}

سهام عبد الفتاح محمد جبر (Les sentiments religieux et mystiques Chez Alphonse de Lamartine) 
Pour bien mettre les points sur les i, il faut souligner l'importance de la corrélation entre les, deux sources; la lumière et la transparence. L'annexion de l'esprit a cette " lumière est accompagnée d'une sensation de lévitation, d'un flottement du corps grâce à la transparence de son esprit. La meilleure combinaison de cette transparence avec la lumière, disons mieux de la perception et de l'absorption de la lumière grâce à la transparence de l'esprit, a été crûment mise en évidence, avec une élucidation qui ne supporte la moindre équivoque:

"Mon âme transparente absorbait la lumière et sereine et brillante avec l'heure et Le lieu d'un élan naturel se soulevait à Dieu. "22

Lamartine ne veut point nous laisser dans le doute. II lui tient à cœur de souligner la transmutation psychologique de son état d'âme pour s'élancer dans le monde de la lumière. Grâce à la transparence de son esprit et son aptitude d'absorber la lumière, il trouve le chemin de son transport mystique extatique. On relève bien l'adjectif "extatique" car l'âme humaine n'est pas faite pour le malheur ; Le seul critère qui détermine l'escapade psychologique d'un malheureux vers les rives de la sécurité, c'est la qualité et la maturité de ses sens spirituels. Combien se font-ils de plus en plus misérables, ceux qui n'ont pas su choisir la voie adéquate de leur affranchissement. II y a plusieurs moyens d'émancipation mais qui ne font qu'empirer peut-être cet état d'âme. Quant à l'élite des âmes, le Créateur veut ressusciter pour une raison qui nous dépasse et que lui seul connaît; il les couvre d'un blindage invincible: celui de la transparence. C'est cette transparence de l'âme, dont a été nanti Lamartine, qui fut son tremplin hors pair vers la voie du Seigneur. Grâce à elle, l'âme absorbe tout ce qui est transparent lumière, éther .

Lamartine a bien décrit ce transfert du monde terrestre à celui ou n'existe que la lumière et tout ce qui est clair et limpide, en faisant le parallèle entre le corps et l'esprit: 
"Je ressemble, Seigneur, au globe de la nuit, qui dans la route obscure ou ton doigt Le conduit, réfléchit d'un cote les clartés éternelles et de l'autre est plonge dans les ombres mortelles. L'homme est Le point fatal ou les deux infinis par la toute puissance ont été réunis." 23

\section{La liberté de l'esprit.}

Cette union de l'esprit et de la lumière représente la concrétisation de la liberté, du libre élan loin. Des humains et de la terre, tout près de Celui qui offre la vie avec ses pures caractéristiques, surtout pour une âme qui cherche à se purifier, pour mériter la fin qui lui revient, après une longue trajectoire faite de périls et de malheurs. Dans ces moments de limpidité, l'âme échappe à la fascination des sens malgré leur existence en les dominant et en maîtrisant leur élan. Et voilà que le corps dégagé de tout poids, est nanti d'une force quasiment surnaturelle. C'est cette force exceptionnelle de l'esprit qui lui permet son extirpation de sa coquille charnelle pour rejoindre les rayons du Cosmos et flotter avec eux du même rythme et de la même cadence. Lamartine nous a fait revivre amplement ces moments sublimes de sa vie :

"Que mon âme à mes sens échappant quelquefois

de son corps détache ne sent pas plus le poids

que le cygne, essayant son aile déjà forte

ne sent le poids léger de l'aile qui le porte.

J'aime dans ce silence à me laisser bercer

à ne me sentir plus ni vivre ni penser

à croire que l'esprit qu'en vain le corps rappelle

à quitte sans retour l'enveloppe mortelle

et nage pour jamais dans les rayons du ciel." 24

La, il participera de cette lumière. Il retrouve sa propre identité dans les paroles adressées à un poète exile:

سهام عبد الفتاح محمد جبر (Les sentiments religieux et mystiques Chez Alphonse de Lamartine) 
"Mon âme est un rayon de lumière et d'amour qui du foyer divin détache pour un jour de désirs dévorants loin de toi consumée, brûle de remonter à sa source enflammée." 25

Dans cette phase ou le corps outrepasse ses limites charnelles et laisse à l'esprit toute l'ampleur de son déploiement, l'esprit est envahi de cette aisance dont il était démuni sur terre. Là, dans ce transport mystique, c'est la liberté qui prend le dessus pas de heurts avec les habitants de la terre; nulle collision avec les obstacles persistants de la vie. La montée vers le trône du Seigneur se fait sans la moindre difficulté. Les portes sont largement ouvertes pour accueillir ces âmes pures qui ont accepté volontiers, toute privation pour s'acheminer vers la voie du tout puissant et savourer le fruit de leur obéissance dans sa forme la plus majestueuse: celle de la liberté:

"Il monte et l'horizon grandit à chaque instant il monte et devant lui l'immensité s'étend comme sous Le regard d'une nouvelle aurore un monde à chaque pas pour ses yeux semble éclore jusqu'au sommet suprême ou son oil enchante s'empare de l'espace et plane en liberté" 26

\section{Le côté éthéré de ce transport mystique:}

Pendant le flottement de l'esprit, le corps perd tout à fait la sensation de sa pesanteur sur terre et se dégage de tout son poids . L'être humain ne se sent plus une créature terrestre mais une entité éthérée qui fait partie d'un autre monde. II ne peut acquérir cette faculté sublime qu'en se détachant de tout lien terrestre et en bridant l'élan de ses besoins sensuels.

Ne peut accéder à cette épuration, que celui qui est obligé d'assumer le poids de sa masse charnelle qui lui est infligée.

سهام عبد الفتاح محمد جبر (Les sentiments religieux et mystiques Chez Alphonse de Lamartine) 
Ces mystiques ne veulent point sentir leur participation à l'existence terrestre que par le volume de leur corps. Lamartine a profondément ressenti ces sentiments. Le désir de s 'affranchir et de se débarrasser des exigences de la chair ne la jamais quitte. Son seul attachement était porte à ce monde d'éther qui délivre l'âme de ses souffrances terrestres, particulièrement pour lui, accable par de tant de malheurs dans sa vie. Nul ne peut mieux apprécier ce transport mystique, a sa juste valeur, que celui qui a éprouvé son besoin urgent, alors l'âme devient saine et sauve, dans son enveloppe de limpidité et de transparence, imprégnée d'une vague sensation de sommeil paisible exempt de tout cauchemar tracassant. Ce qui est remarquable chez Lamartine, dans cet état de flottement, c'est que l'effort est radicalement supprimé et laisse la lévitation apparaître immobile. Dans Jocelyn, Lamartine a bien accusé ce phénomène merveilleux:

"Et l'âme qui s'endort

nage dans l'infini sans ailes sans effort, sans soutenir son vol sur aucune pensée. Mais immobile et morte et vaguement bercée, avec ce sentiment qu'on éprouve en rêvant qu'un tourbillon d'été vous porte et que, Le vent vous prêtant un moment ses impalpables ailes vous planez dans l'éther tout semé d'étincelles."27

\section{L'Energie et l'Enthousiasme de la foi.}

L'énergie de ces transports mystiques est la force spirituelle de la foi chez Lamartine. Pour s'élancer dans le monde de la lumière et de la transparence, il nous a suffisamment démontré le déclencheur spirituel de sa superbe envolée transcendante ; c'est un enthousiasme intérieur qui amorce la transmutation du monde des sens à celui de l'esprit :

"Tantôt soulevé par l'enthousiasme intérieur qui 
me dévorait, courant sur les bruyères comme porte par un esprit qui empêchait mes pieds de toucher le sol." 28

Cette énergie de l'âme est dotée d'une force révélatrice et d'un entrain hors pair. Elle représente la source d'exaltation la plus efficace qui puisse exister, capable de lancer Lamartine dans ses spirales de lumière. Cette force qui s'infiltre dans les minimes particules de l'être lamartinien, lui inculque son dynamisme spirituel capable de l'emporter

Dans ses vers adressés à L'Abbé de Lamennais, Lamartine nous révèle ce don exceptionnel. Dont il a largement cueilli les fruits:

"Aux pures régions où j'aime à m'envoler

l'enthousiasme aussi vient me la révéler lui seul est mon flambeau dans cette nuit profonde

et mieux que la raison, il m'explique Le monde.

Viens donc, il est mon guide, et je veux t'en servir

A ses ailes de feu, viens laisse-toi ravir." 29

Cette énergie intarissable qui anime sa capacité véhémente, de forcer les ténèbres n'est autre que le sue d'une foi inextinguible. Toute la force de son âme et l'énergie de son esprit sont le fruit d'une foi inébranlable qui lui a permis d'escalader les hautes régions de l'éther en perçant les voiles le plus impénétrables:

"Comme l'oiseau qui voit dons les ombres funèbres

la foi, cet ail de l'âme, a percé mes ténèbres son prophétique instinct m'a révélé mon sort." 30

\section{L'absence du temps et de l'espace}

Le temps et l'espace n'existent pas dans le glossaire des mystiques. Ces notions sont radicalement bannies de leur perception. Amalgamés avec leur monde de transparence, ces spirituels ne connaissent que le sens de la paix et de la sérénité.

سهام عبد الفتاح محمد جبر (Les sentiments religieux et mystiques Chez Alphonse de Lamartine) 
Dans leur transport spirituel ils échappent à la portée du temps et de 1'espace. Leur orbite est illimitée et dépourvue de tout horizon tant que leur esprit est globalement dissous dans leur "Havre de Paix ". Grâce à cette faveur, ils touchent de près la "Vérité" éternelle. Dans ses vers adressés à L'Abbé de Lamennais, cette notion est mise en relief:

"Déjà l'ombre du monde, à nos regards s'efface;

Nous échappons au temps, nous franchissons l'espace

$E t$, dans l'ordre éternel de la réalité

Nous voilà face à face avec la vérité!" 31

Cette disparition de l'effet du temps et de l'espace est, en effet une sorte d'oubli de l'existence terrestre dont les seuls critères sont la succession des jours et Le changement des lieux. Ailleurs, ou l'élan mystique emporte ses favoris; ils ne connaissent que la pérennité d'une dissolution totale dans l'essence divine, un flottement sans répit dans l'éther et la lumière. Lamartine a décrit éloquemment cette symbiose bénéfique avec l'univers et ses invisibles molécules:

"C'est toi! c'est moi ! je suis! j'adore
Le temps, l'espace s'évapore
J'oublie et l'univers et moi!
Mais cette ivresse de l'extase
Mais ce feu sacre qui m'embrase
Mais ce poids divin qui m'écrase
c'est toi, mon Dieu, c'est encore toi!" 32

Grâce à cette énergie tonifiante de l'âme dans ces moments indicibles de dissolution totale avec le Cosmos, les mystiques peuvent contacter les apparitions célestes des âmes purifiées. Les adeptes de cette vie spirituelle profitent d'inestimables instants de sérénité et de bien être loin des problèmes de notre

سهام عبد الفتاح محمد جبر (Les sentiments religieux et mystiques Chez Alphonse de Lamartine) 
vie terrestre. Cette énergie et cet enthousiasme permettent à l'esprit de jouir d'un dynamisme hors pair et d'une chaleur prégnante. Alors, cette lumière enveloppe l'esprit de ces mystiques et répand la chaleur dans leur âme, ce qui dissipe toutes les incertitudes qui peuvent gagner leur âme. Tout état de perdition psychologique fuit à cette énergie. Grâce à la tiédeur du courant spirituel, la vie devient aussi limpide que l'onde qui l'envahit. C'est en évoquant la disparition de Madame la Duchesse de Broglie que nous saisissons la valeur génératrice de cette lumière comparée à celle du "feu":

\section{"Apparitions célestes, (...) feux que notre nuit voit poindre Oh! Mourons pour les rejoindre." 33}

\section{Le côté olfactif: une senteur agréable.}

Le transport mystique n'est pas uniquement accompagne d'une apparence lumineuse et éthérée, il y a le facteur olfactif qui intervient. Les délices de cet enthousiasme ne sont pas purement engendrées d'éclairs et d'étincelles, il y a l'appréciation psychologique d'une senteur douce et agréable. Cet éther n'est point fastidieux et déconcertent comme peu, se l'imaginer un matérialiste. Loin de là, car il est chargé d'un parfum qui flotte et chatouille l'odeur de ceux qui ont éprouvé cet état psychologique. Un monde tout à fait envoûtant et réconfortant pour les adeptes du mysticisme. En revanche, il apparaîtra absolument fictif pour les profanes et ceux qui n'ont pas traverse ce tunnel de l'esprit et ses phases majestueuses.

Une sorte de sensation olfactive attrayante pour ceux qui ont renoncé aux faux plaisirs de 1 'existence pour aspirer à une spiritualité capable de procurer l'ataraxie de l'âme et la sérénité de l'esprit. En méritant la montée sur le podium de ce monde 
éthérée, l'on se transfère dans un univers habité par les Saints et les bien- heureux qui l'ont vaillamment mérité, à force d'abnégation et de sacrifice. Cette élite des âmes peut savourer à sa guise l'émanation du parfum et la lumière qui s'y dégagent dans les moments de haute extase mystique. Lamartine soulignera , ce trait fondamental en évoquant la mort de Mme la Duchesse de Broglie:

"La, ces âmes fugitives
(...) qui traversent nos ténèbres
en y jetant leur éclair
la, ces enfants et ces femmes
toute cette fleur des âmes
qui laisse un parfum dans l'air." 34

\section{La Science et la paix.}

Pour l'équilibre des choses, la justice divine qui sait si bien le poids du sacrifice de ces béats, leur permet l'accès à la "Vérité": véritable Science divine. Elle inculque dans leur âme passionnée d'Infini, la beauté et la lumière d'une Science puisée dans ce "Haut" séjour. En échange de cette abnégation sur terre, il y aura la Science de la Vérité, authentique science de l'esprit qui n'est révélé qu'à ceux qui l'ont méritée: Dans ce monde fascinant de l'esprit. On pourra puiser la véritable nourriture de l'esprit. II y a l'auréole de la beauté qui entoure cette science, à la portée de chaque aspirant à cette source divine. Les portes y sont grandement ouvertes, à chaque habitant de la terre de faire le premier pas pour découvrir ce paradis de l'esprit:

"Où l'on puise à jamais la, science et l'amour où dans des océans de beauté, de lumière l'homme altère toujours, toujours se désaltère. "35

Dans cet enthousiasme mystique, Lamartine déborde de bénédiction et de reconnaissance. Pendant ces moments de 
clairvoyance, il exprime tout ce qu'il détient de gratitude, tout ce sublime état d'âme n'est que l'expression d'une paix intérieure et d'une sérénité extatique. II sera capable alors, de lancer un défit, outrancier a toutes les normes de la confiance, contre ce qu'appellent les mortels "la Mort"; même si les vers rongeurs Le menaceront par leur présence:

"Je bénissais Celui dont l'immense nature

prête place au soleil à chaque créature

et" la terre de Dieu, qui du val au coteau

a pour nous cacher tous un coin de son manteau

et je ne savais pas dans ma paisible extase

si quelque ver rongeur piquait au cour ma phrase." 36

Malgré la griserie de cette ataraxie mystique qui anime la vie de Lamartine, il ne perd point de vue que c'est grâce au Tout-Puissant qu'il doit sa jovialité. II appréciera toujours, avec reconnaissance la récompense de son stoïcisme exemplaire:

"Tu rends cent mille fois ce qu'on te sacrifie." 37

\section{Le mysticisme religieux et l' amour}

L'amour est la plus grande expression du Mysticisme chez Lamartine il est le langage divin que seuls peuvent communiquer les cœurs purs et transparents. C'est la meilleure faculté dont peuvent être dotes les habitants de la terre pour percevoir le langage des âmes et l'expression des cœurs. Son "Code" n'est qu'une simple palpitation, et une légère vibration que seul peut mesurer Celui qui l'a constituée. Cette faculté d'échange avec tout être qui possède un cœur palpitant s'avère le meilleur centre émetteur de la spiritualité. II capte dans les ondes tout ce que possède les vivants et les non-vivants de sublime, de frétillant qui ruisselle avec le sang dans leurs veines-Excellent interprète, ultra-sensible ou divin, il est

سهام عبد القتاح محمد جبر (Les sentiments religieux et mystiques Chez Alphonse de Lamartine) 
toujours prêt à comprendre et à écouter attentivement le langage de la prière:

"Ce langage senti, touche, illumine, enflamme de ce que l'âme éprouve interprètes brûlants il n'a que des soupirs, des ardeurs, des élans c'est la langue du ciel que parle la prière et que le tendre amour comprend seul sur la terre." 38

C'est Le fruit d'une âme pure et tout ce qu'il y a de sublime et de substantiel qui se dégage d'un esprit pétri dans l'Univers Providentiel. II s'exhale du divin que l'homme a en soi pour revenir à la source qu'il a crée après avoir parcouru le long périple de la vie et répandu cette essence divine sur celui qui la réclame de nos frères et sœurs sur la terre. II aspire toujours a s'élever. Rien n'est plus fort, plus étendu, plus parfait que l'amour parce qu'il est né du divin et ne peut se reposer qu'en Dieu. La prière est son chemin le plus court qui amène à Dieu. Cette prière, Lamartine l'a toujours sollicitée car il sait qu'elle ne lui refuse rien. Le poète est Le premier à savoir que la prière ouvre les portes du miracle: c'est elle, avec tout-ce qu'elle implique de senteur agréable, qui est capable de réunir les cœurs pour en faire une force invincible et irrésistible devant l'usure du temps vers, un au-delà spacieux et accueillant:

"Ah! s'il en est, doux souffles de l'aurore emportez-nous avec l'encens des fleurs emportez-nous où les, âmes sont sœurs!

Nous prierons mieux le Dieu que l'astre adore car l'âme aussi veut le ciel pour éclore et la prière est le parfum des cœurs!" 39

Ce mysticisme embrasé ne cessera jamais de déployer ses ailes sur ceux qui le prennent pour abri contre les intempéries de la vie. Son effet bénéfique est démesuré. L'ampleur de ses avantages échappe à tout inventaire. II y aura toujours quelque chose à récolter tellement son champ est vaste. Ne suffit- il pas

سهام عبد الفتاح محمد جبر (Les sentiments religieux et mystiques Chez Alphonse de Lamartine) 
de posséder sa chaleur intarissable et inépuisable animant le flot du sang dans nos veines? Alors tous nos instincts sont purifies et le divin commence à nous revêtir par sa splendeur et sa fascination. Cette pureté mystique se dévoile sans ambages, ni détour dans les sentiments de Jocelyn vis à vis de Laurence:

"Aussi depuis qu'un cour bat enfin sur Le mien tous mes instincts sont purs et me portent au bien Mon âme qui souvent tarit dans la prière nage toujours en moi dans les flots de lumière une telle clarté m'échauffe dans ses yeux le timbre de sa voix n'est si mélodieux

tant de divinité sur ce doux front rayonne que la splendeur de Dieu jour et nuit m'environne." 40

Le Ciel ne connaît point la sordidité. Sa générosité et sa clémence offre à tous les êtres le saveur de l'amour indispensable à leur subsistance:

"Aux uns c'est un sort triomphant

à ceux-ci Le cour d'une femme

à ceux-là Le front d'un enfant

(...) chaque ruche d'homme a son nid." 41

Quant à Lamartine véritable érudit de la science divine, il savait parfaitement tirer parti de ce lot d'Amour. II n'est pas averti pour rien puisqu'il se réserve la source même de l'Amour:

"Ah; livre a leur soif assouvie

cette eau des sources de la vie!

Mais ma source a moi n'est qu'au ciel." 42

Lamartine préférait l'Amour divin, l'Amour pur et sublime à celui des sens débridés. Mais à quel prix il préservera cette préminence! Quelle était véhémente cette lutte sans merci, et combien fut pénible cette résistance à l'appât de la 
chair! L'amour chez Lamartine c'est l'amour en Dieu, même dans sa vie conjugale, il ne se marierait que pour plaire à Dieu.

Qu'il le veuille ou non, son corps avec ses exigences n'a pas encore disparu, il en est bien conscient. Mais ce n'est pas lui, cet implacable mystique qui laissera les sens étouffer la saveur du feu, divin qui anime son corps. Grâce à une volonté d'acier et à une ferveur qui puise son étincelle d' ailleurs, il surmontera les vagues, de l'appel sensuel:

"Des que j'aimais, je rougis de ces profanations de la poésie aux sensualités grossières, l'amour fut pour moi le charbon de feu qui brûle mais .qui purifie les lèvres. Je pris un jour mes deux volumes d'élégies, je les relus avec un profond mépris de moi-même, je demandai pardon à Dieu du temps que j'avais perdu a les écrire, je les jetai au brasier, je les regardai noircir et se tordre avec leur belle reliure de maroquin vert sans regret $n i$ pitié et j'en vis monter la fumée comme celle d'un sacrifice de bonne odeur à Dieu et au véritable Amour.' 43

Lamartine, cet homme endurci par les épreuves, pétri par le malheur qui l'a foudroyé sans le démolir. Il est persuadé que la moindre vacillation, le simple fléchissement aux "pauvretés sensuelles" c'est la privation du secours de ces "ailes" qui amènent les âmes pures vers le seuil du trône divin:

"A travers l'infini, sur l'aile de l'amour

(...) et jusqu'à Dieu lui-même arrivant éperdues." 44

Oui, cette agréable fleur de l'amour ne s'obtient pas facilement, il faut savoir brider ses désirs et dominer ses instincts pour que puisse se propager la senteur de l'amour:

"Etendre son esprit, resserrer ses désirs

c'est là ce grand secret ignore du vulgaire." 45

سهام عبد الفتاح محمد جبر (Les sentiments religieux et mystiques Chez Alphonse de Lamartine) 
Admirable spirituel, expert de la science divine; Lamartine a réussi à trouver le chemin de l'Amour; il a compris que parfois ce qui peut être pénible à l'âme est capable de lui procurer son bonheur. II suffit de savoir exploiter les dons exceptionnels de son mysticisme et son indifférence à la vie; le venin n'est-il pas parfois la 'base d'une cure miraculeuse?

"L'amour est innocent quand la vertu l'allume

(...) la constance l'honore et le malheur l'épure. ${ }^{46}$

L'énergie de l'amour spirituel, ne connaît point de bornes. Emanant du suc divin qui arrose les veines du mystique, elle lui offre tout Le dynamisme nécessaire pour le transporter au Ciel de la pureté loin des soucis et du miasme d'un matérialisme suffoquant:

\section{"Pour cueillir cet amour, fruit immatériel \\ chacun de mes soupirs m'enleva vers le Ciel quand elle disparut derrière le nuage mon cour purifie contenait son image et je ne pouvais plus de peur de la ternir redescendre jamais d'un si haute souvenir;" 47}

Car pour échapper au néant de l'existence, à sa fadeur, il faut s'agripper à l'Amour, ce noble guide capable de forcer les murs des ténèbres:

"L'homme est néant mon Dieu, mais ce néant t'adore il s'élève par son amour." 48

Cela n'est point le seul lot du gagnant, il n'y a pas que le sublime, il y a la paix et la certitude que peut prouver l'amour :

"Comme la vague orageuse

s'apaise en touchant Le bord

سهام عبد الفتاح محمد جبر (Les sentiments religieux et mystiques Chez Alphonse de Lamartine) 
comme la nef voyageuse

s'abrite a l'ombre du port

comme l'errante hirondelle

fuit sous l'aile maternelle

l'oil dévorant du vautour

à tes pieds quand elle arrive

l'âme errante et fugitive

se recueille en ton amour." 49

L'Amour ne cessera jamais de nous ébahir par ses recettes miraculeuses il est capable de transpercer les nuits par la lueur de ses ailes, aussi est-il apte d'ensevelir tous les péchés et rompre la morosité des regrets et du remords. Lamartine est bien avise sur le droit au pardon que donne l'amour. Il n'implorera le Seigneur qu'en s'assurant de la présence de l'Amour dans la vie de ceux qui l'ont quitte :

"Etends sur eux la main de ta clémence

Ils ont péché mais le ciel est un don

Ils ont souffert, c'est une autre innocence

Ils ont aimé, c'est le sceau du pardon." 50

\section{L'Amitié et le mysticisme religieux}

Ce mysticisme ardent n'est pas vain ni fortuit. Ses récoltes se font aussi bien dans notre monde qu'après la vie. A ceux qui l'ont conquis il ouvre les portes de l'amitié. Chez Le poète, l'amour et l'amitié ont le même élan sous deux noms distincts. En effet, Lamartine se retrouve et happe sa propre existence ou le divin occupe sa place prépondérante. Les plus vives, les plus délicates impressions sont celles qu'éveille ce sentiment de l'amitié, c'est que celle-ci participe plus que tout autre sentiment au rythme universel. La apparaît la profondeur, l'authenticité d'une similitude qui provient de la même Source

سهام عبد القناح محمد جبر (Les sentiments religieux et mystiques Chez Alphonse de Lamartine) 
et qui réunit deux amis. L'Amitié n'est que la cristallisation de l'oeuvre divine sur la terre. La nature entière cherche son bonheur dans ce dédoublement, aussi le poète se complait-il a retrouver dans l'univers ces attaches qui s'établissent entre deux êtres car l'ami;

"C'est Le rayon du ciel par l'eau répercuité qui remonte au rayon pour doubler sa clarté, c'est le son qui revient de l'écho qui répète seconde et même voix à la voix qui Le jette c'est l'ombre qu'avec nous le soleil voit marcher sœur du corps qu'à nos pas on ne peut arracher." 51

L'amitié devient par la noblesse de ses impulsions, l'élan même de toute âme vers l'amour divin. Lamartine n'a pas louvoyé pour mettre en relief le côté mystique de l'amitié. La lettre du 10 juin 1808 à son ami Guichard de Bienassis en est le témoin :

"Qu'est-ce que l'amitié? Le lien de deux cours

qu'unissent la vertu, les goûts et les humeurs.

Divin attachement, ne de la sympathie

que le sentiment forme et le temps fortifie." 52

Le poète n'oubliera pas de battre en brèche la fausse amitié connue par les intéresses: Il attaquera ces raisonneurs entêtés qui ne voient dans leur fausse amitié que la recherche de l'intérêt:

"Halte-la,! diras-tu raisonneur entêté

Oh! dépouille une erreur ou ton cour t'a jeté.

L'amitié n'est plus qu'un nom, qu'une faiblesse impure fille de l'intérêt et non de la nature." 53

سهام عبد الفتاح محمد جبر (Les sentiments religieux et mystiques Chez Alphonse de Lamartine) 
L'amitié doit être purement désintéressée, elle sera fonde sur l'amour divin réciproque qui unira les êtres humains sur la terre: pour Dieu et en Dieu car Celui qui a créé les âmes doit seul les unir. Cela sera l'idiome de Lamartine;

"Je suis sûr d'un ami dans tout homme qui prie." 54

Le poète ne sépare pas l'amour de l'amitié, ils sont les deux branches de l'arbre divin. Le paroxysme de ces deux tendances se réalise par leur capacité d'atteindre les "hauteurs" de la sincérité ou les esprits se rencontrent et se confondent; la réside la forme exhaustive de ce sentiment mystique:

"L'amour et l'amitié ne sont au fond que l'image d'un être réciproquement entrevue et doublée dans le cour d'un autre être. Quand ces deux images se confondent tellement que les deux n'en font-plus ou' une, l'amitié ou l'amour sont complets." 55

Que de privilèges peut-on acquérir de cette sainte loi qui régit les esprits purs. Combien sont grandioses les avantages de cette union divine! N'est-elle pas le secret de notre vie sur terre, l'élixir et le sérum qui détruisent tous les maux de l'humanité:

"Dieu qui a créé l'amitié pour faire supporter la terre." 56

Il ne cherche que le salut de l'humanité. La vitalité des cours sains ne découle que de la véracité des sentiments, de leur sincérité :

"L'amitié, ce soleil de l'âme

me ranima de sa chaleur

fond ma neige à sa tiède flamme

et me rend le printemps du cour." 57

سهام عبد الفتاح محمد جبر (Les sentiments religieux et mystiques Chez Alphonse de Lamartine) 
Et quel remède que celui de l'Amitié contre les ouragans de la vie; Lamartine n'oubliera jamais cette leçon apprise de ses amis les orientaux: "Les orientaux qui ont tout dit parce qu'ils ont tout senti les premiers, ont un proverbe plein de ce sens exquis de l'amitié:

"Pourquoi Dieu, disent-ils, a-t-il donne une ombre au corps de l'homme? C'est pour qu'en traversant le désert l'homme puisse reposer ses regards sur cette ombre, et que le sable ne lui brûle pas Les yeux." 58

L'amitié ne doit pas régner uniquement entre quelques personnes, elle doit s'étendre sur tous les peuples car elle est le seul emblème qui peut réunir les fils de la terre. La couleur de la peau n'est qu'un teint artificiel de l'humanité, la véritable essence de tous les êtres humains est la similitude de l'esprit, l'homogénéité du noyau qui est l'esprit doit être le seul facteur qui unit les humains sur une base bien fondée et ou sont; bannis la haine et l'égoïsme d'une monstrueuse discrimination raciale :

"Et pourquoi nous haïr et mettre entre les races

Ces bornes ou ces eaux qu'adhère l'oil de Dieu? ${ }^{59}$

\section{Le Mysticisme religieux et la notion de la patrie:-}

L'esprit mystique de Lamartine évalue la notion de la "patrie" qui la lie à Dieu. Dans ses transports mystiques il ne trouve aucun barrage, aucune digue ni rivière qui le sépare de Dieu, pourquoi donc faisons-nous de ces fausses barrières un obstacle entre les peuples de la terre?

"Ce ne sont plus des mers, des degréss, des rivières qui bornent l'héritage entre l'humanité

سهام عبد الفتاح محمد جبر (Les sentiments religieux et mystiques Chez Alphonse de Lamartine) 
Les bornes des esprits sont leurs seules frontières. " 60

Un esprit éclairé par la lumière divine ne mettra aucune digue entre lui et les autres sur la terre, car :

"Le monde en s'éclairant s'élève à l'unité". ${ }^{61}$

C'est la subtilité de l'intelligence qui détermine l'ampleur de chaque esprit et sa conception de la fraternité entre les peuples de la terre; Les esprits bornes seuls se renferment dans leurs ténèbres. Quant a Lamartine, il est parfaitement réceptif à chaque âme qui existe dans notre monde surtout celle qui pense. Sa conception de la fraternité et de l'amitié est nette et claire puisque sa patrie;
"(...) est part out ou rayonne la France, ou son génie éclate aux regards éblouis!
"Chacun est du climat. de son intelligence.
Je suis concitoyen. de toute âme qui pense.
La vérité c'est mon pays." 62

Le poète souhaite que ses sensations mystiques gagnent toute l'humanité, il ne voit les choses que par le truchement d'une spiritualité ardente prête à aider tous les êtres humains. Il ne désire que:

$$
\begin{aligned}
& \text { "Fondre les nations en peuple fraternel } \\
& 63 \text { marqué au front par Dieu de son chiffre éternel." }
\end{aligned}
$$

Lamartine déplore l'ingratitude de l'humanité, le reniement de ses origines. Ne sommes nous pas nés d'un seul père et d'une seule mère? Nous devons tous, peuples de la terre, revenir 'a la

سهام عبد الفتاح محمد جبر (Les sentiments religieux et mystiques Chez Alphonse de Lamartine) 
source. Nul obstacle n'existait entre nos plus lointains ancêtres, pourquoi les créerons-nous de nos jours? Allons-tous appliquer les lois de l'amitié.

\author{
"Cette loi qui dit à tous ".Frères" \\ a brise ces divisions \\ qui séparaient les fils du père \\ en royaume et nations \\ semblable au métal de Corinthe \\ qui, pendant la forme et l'empreinte \\ du sol ou du rocher natal \\ quand sa lave fut refroidie \\ au creuset du grand incendie \\ fut fonder dans un seul métal. ${ }^{64}$
}

Véritable leçon donnée par un fervent mystique qui a su rendre à L'amitié la place qui lui revient grâce à son regard scrutateur dans les plus profondes souches de l'humanité, il a bien expliqué l'unité originelle de l'esprit. Le capacité d'un esprit de capter dans son élan spirituel, tous les cœurs acquis par L'amitié, est le meilleur couronnement du mysticisme. Il le dira volontiers dans ses vers adresses à M. Bouchard en 1837:
"Servons l'humanité, le siècle, la patrie vivre en tout, c'est vivre cent fois c'est vivre en Dieu, c'est vivre avec l'immense vie qu'avec l'être et les temps sa vertu multiplie rayonnement lointain de sa divinité." 65

Polariser les cœurs, c'est intégrer à son propre esprit celui des autres pour constituer un cortège rayonnant et solennel prêt à se diriger vers le trône du Seigneur et s'y confondre:

سهام عبد القناح محمد جبر (Les sentiments religieux et mystiques Chez Alphonse de Lamartine) 
"C'est tout porter en soi comme l'âme suprême qui sent dans ce qui vit et vit dans ce qu'elle aime et d'un seul point du temps c'est se fondre soimême dans l'Universelle Unité. ${ }^{66}$

Lamartine a parfaitement connu des sa prime jeunesse que Dieu est la meilleure patrie de tous les hommes. La puissance divine est capable de réunir ceux que les nouvelles sociétés éloignent par un simple trait de crayon ou une carte géographique:

\section{"Ah! de tout ce qui s'aime et de tout ce qui prie la présence est en Dieu, car Dieu c'est leur patrie. ${ }^{67}$}

Secrets majestueux d'un monde lumineux ou toute l'humanité peut trouver ses remèdes et son élixir. Chaleur d'un Universel foyer ou tous les déshérites peuvent combler la vacuité creusée par cette vie. Cela était le rêve fascinant de ce grand poète mystique. L'optimisme ne quittera pas Lamartine car il connaît que chaque réalité avait comme origine un simple rêve, une conception banale. Il ne trouve pas de raisons pour que le doute rode autour de ses convictions purement spirituelles et transcendantes. Il est sûr que la primaire inextinguible est capable de faire des habitants de ce globe, une seule famille:

"Je jette à mon Dieu mon âme et je me dis en lui

j'ai les eaux de ma soif, la faim de mon ennui

j'ai l'âme dont le cour de tout amour abonde

la famille immortelle et l'invisible monde," 68

C'est l'Etre Suprême qui a créé cet univers, l'a couvert de son firmament, tout le monde doit sentir la paix et la fraternité sous ce toit céleste. Les liaisons humaines doivent y régner, les races

سهام عبد القناح محمد جبر (Les sentiments religieux et mystiques Chez Alphonse de Lamartine) 
s'entendre, s'aimer mutuellement, à celle qui jouit d'un meilleur sort de tendre la main aux autres:

"Vous n'établirez pas ces séparations

en races, en tribus, peuples ou nations

et quand on vous dira cette race est barbare

ce fleuve vous limite" ou "ce mont vous sépare"

dites "Le même Dieu nous voit et nous bénit,

Le firmament nous couvre et Le ciel nous unit." 69

Notre tâche sur cette terre doit être fructueuse et florissante, notre amour global. La part de considération et de fidélité due à tout être de notre planète est un facteur indispensable pour l'épanouissement de l'humanité. La considération réservée aux autres est le premier élément d'une société universelle unifiée, ou seuls les meilleurs" préceptes règnent. Il faut préserver la dignité de ses frères, c'est le seul moyen de protéger la notre :

"Avilir les humains, ce n'est pas se grandir
c'est éteindre Le feu dont on veut resplendir
c'est abaisser sous soi Le sommet ou l'on monte
c'est sculpter sa statue avec un bloc de honte." 70

Véritable leçon d'amour et solide édifice spirituel fonde sur une base inébranlable. Un amour débordant qui préserve l'estime des autres et une amitié exemplaire d'un grand cœur. Grâce à la bravoure de son esprit, il a renie l'outrecuidance de l'index de sa vie; car pour lui:

"tout est intéressant, tout est important, tout est respectable dans les destinées du plus obscur et du plus insignifiant de tous les êtres." 71 
Reconnaître aux autres leur juste valeur, sans toutefois se sous-estimer est l'expression d'un esprit prêt à s'entraider avec les autres. C'est le premier pas d'un esprit qui s'apprête à nier la vanité de la vie pour s'amalgamer avec l'Entité divine. Tel était le chemin poursuivi par ce grand esprit en parlant de Pétrarque. Avec une humilité surprenante et une résignation singulière il dira:

"Tout ce que j 'ai écrit de vers ne vaut pas un sonnet parfait de Pétrarque (...) Les hommes ont des hasards de célébrité comme les lieux voilà! Ils ne doivent ni s'enorgueillir de leur succès, ni s'humilier de leur revers, mais faire de leur mieux dans tous les temps et s'en rapporter de leurs ouvres à leurs ouvres, plus qu'à la renommée: ${ }^{72}$

Lamartine répugnait fortement à la prétention et à la vantardise. La simplicité de son caractère, sa philanthropie avait horreur de toute obstination dans la fierté. Entendons-le dénoncer ces graves défauts du caractère, à propos du livre de Corinne de Mme de Staël :

"Je le lus en deux jours, me croyant transporte dans un monde idéale naturel, poétique oppose en tout a cette aride et froide société, a ce monde si ridicule et si fier dans ses idées, si despotique et si mort dans ses opinions a des complots de coterie qui font toutes mes peines et mes obstacles." 73

L'amour combat rigoureusement l'égoïsme et prêche l'altruisme qui réalise la perfection de l'esprit, car l'altruisme 
seul peut atteindre les cimes du parfait par l'amour d'autrui. Aimer son prochain, c'est évoluer sur l'échelle spirituelle en unifiant davantage les cœurs aux nôtres jusqu'à l'atteinte de l'indivisible unité, puisqu'

"au lieu de resserrer son âme

L'homme: Immense en étend la trame

aussi que l'humanité

et sur de grandir avec elle

répand sa vie universelle

dans l'indivisible unité." 74

La philanthropie bride les tendances expansionnistes des peuples et entraîne la maîtrise de soi et de ses besoins. C'est en aimant nos frères que l'on apprend à respecter leur territoire et leurs propriétés et de la sorte, la paix régnera facilement dans le monde. Lamartine détestait les guerres, ce "meurtre par milliers" que l'on appelle victoire:

"La guerre, ce grand suicide

ce meurtre impie a mille bras." 75

Le droit de vie ou de mort n'appartient qu'à Dieu seul qui:

$$
\begin{aligned}
& \text { "(...) compte ses gouttes } \\
& \text { et vengeur les retrouve toutes } \\
& \text { où dans la veine .. où sur la main!" } 76
\end{aligned}
$$

Le Cure de Valneige a bien été l'interprète de ce messager d'amour qui respecte chaque goutte de sang de ses frères, même le sang coagule dans les veines après la mort. Cette cure ne se contente pas d'opposer son indignation et sa charité aux

سهام عبد الفتاح محمد جبر (Les sentiments religieux et mystiques Chez Alphonse de Lamartine) 
fanatiques de son village, qui parlent de traiter "comme un chien" Le cadavre du colporteur juif pour la jeter aux crevasses du roc. Ils osent dire que "la croire" ne doit point d'ombre à celui qui la nie et qu'à leurs seules dépouilles est due la terre bénie. Durant ce scandale inhumain Jocelyn fait honte à ces fanatiques de leur dureté d'âme et décidant que la terre bénie sera pour le juif comme pour le fidèle, Le cure de Valneige prend sur lui d'enterrer à L'ombre de La croix ce corps dont les paysans aveugles répétaient que sa seule présence serait aux cadavres des fidèles une insulte et une souillure. Pour vaincre l'esprit belliqueux, on doit oublier ses racines par l'entente universelle, l'amour et l'amitié.

Evoquant les enfants de Caïn dans la "Chute d'un Ange", Lamartine compare la démence de ces familles fugitives à celles des peuples d'aujourd'hui qui haïssent les autres peuples. Le poète s'indigne contre cet esprit de haine qui ne fait qu'éloigner les humains de la vie du Seigneur:

"Ennemis éternels des races étrangères leur brutale équité se bornait à leurs frères

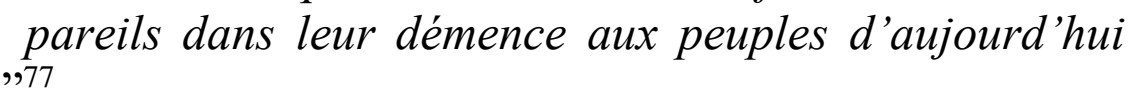

Lamartine a attaqué violemment cet esprit agressif et inhumain, incarne dans le personnage des Titans. Il a battu en brèche leur sadisme et leur soif de sang. Les colonnes de leur palais sont "vivantes", des êtres humains humiliés, les tapis sont des chevelures, les lits et les oreillers sont des corps de femmes. Ils trouvent leur détente dans la souffrance physique et morale de leurs esclaves, parmi d'autres innombrables. C'est l'amitié qui doit régner aussi bien entre les peuples qu'entre les personnes. 
La spiritualité profonde qui a caractérise la vie de Lamartine n'est pas celle du repliement sur soi. Loin de là, elle est d'une effervescence qui répond l'amour dans tous les coins de la terre et qui pénètre chaque être de sa rosée matinale, surtout pour les personnes défavorisées et désemparées:
"Votre ombre ombragera Le passant, votre pain, restera sur le seuil pour quiconque aura faim vous laisserez toujours quelques fruits sur la branche pour que le voyageur vers ses lèvres la penche et vous n'amasserez jamais que pour un temps car la terre pour vous germe chaque printemps et Dieu, qui verse l'onde et fait fleurir ses rives, sait au festin des champs Le nombre des convives," 78

\section{Le mysticisme religieux et la famille}

Ce mysticisme ressuscitera toutes les valeurs sociales en embrasant les feux éteints des foyers. Le poète refuse l'affaiblissement du sentiment humain, base fondamentale d'une vie saine:

\section{"Ainsi pères sans droits, fils sans reconnaissance tout sentiment humain a perdu sa puissance des feux sacres du cour Le foyer éteint." 79}

Ce mysticisme qui fait circuler l'amour dans les veines de Lamartine ne peut pas être considère comme un simple vernis, car il rayonne sur toute la famille. II commence par rendre hommage au père:

سهام عبد الفتاح محمد جبر (Les sentiments religieux et mystiques Chez Alphonse de Lamartine) 
"Sers le jusqu'au tombeau, serviteur sans salaire

d'une piété tendre honore ses vieux ans

ta bénédiction est dans ses cheveux blancs." 80

La reconnaissance et l'obéissance au père sont des devoirs sacres :

"Quand ton père a parlé, sons murmure obéis.

Car, devant Dieu, Le père est au-dessus du fils

C'est de lui que tu liens la vie et la parole." ${ }^{81}$

La reconnaissance à la mère occupe de même un rôle prépondérant dans cet Amour mystique. Les avantages de cette gratitude ne seront point négligeables. C'est en réhabilitant le rôle de la mère que l'on érigera un édifice familial solide capable à son tour de constituer une société épanouie. L'éducation saine de la jeunesse sera la lumière étincelante de l'adolescence. L'enseignement d'une mère reste burine dans les cœurs de cette adolescence:

"Cependant je ne sais quelle horreur du blasphème soit que ce fut l'effet de ce nom du Seigneur que ma mère avait mais comme un sceau sur mon coeur." 82

Aussi Lamartine se montre-t-il aussi bien vigilant qu'attentionné à l'égard de l'équilibre entre tous les individus d'une société. Son sens irréprochable de l'Universel lui a permis de bien propager l'idiome d'une équité et d'un équilibre conjugal. Le règne de la confiance et de la compréhension réciproque est indispensable pour que les relations conjugales soient imperturbables. L'adultère est une grave maladie qui doit être exclue d'une société. Le désir illégitime en soi est condamnable:

سهام عبد الفناح محمد جبر (Les sentiments religieux et mystiques Chez Alphonse de Lamartine) 
"Vous ne porterez pas un désir sur sa femme 83

car la femme de l'homme est son corps et son âme."

Toutes les relations sont inspirées d'un mysticisme ardent, le respect des droits d'autrui n'est pas seulement l'apanage des êtres humains, car Lamartine n'a pas oublié les autres créatures:

\section{"La chaîne a mille anneaux va de l'homme à l'insecte que ce soit le premier, le dernier, le milieu n'insultez aucun car tous tiennent à Dieu.', 84}

L'exhalaison de l'Amour qui tonne dons les entrailles de Lamartine était équitable dans sa répartition. Les brutes aussi ont droit à ce suc divin. Le poète nous incitera à les aimer; avec une sincérité touchante, il proposera son conseil:

\section{"Vous ferez alliance avec les brutes même car Dieu qui les créa veut que l'homme les aime d'intelligence et d'âme a différents degrés elles ont eu leur part, vous la reconnaîtrez." 85}

La plus haute expression de ce pacte d'amour, ne prédit pas de théories superflues ni d'allusions frivoles nous conseille les principes fondamentaux de la véritable essence humaine. Il faut procéder à la pitié et la tendresse avec ces êtres que Dieu a créés dans leur condition inférieure". C'est une lutte de conscience sans merci au quel nous serons affrontes pour faire preuve de notre qualité humaine. Ce n'est que par l'acuité de notre sensibilité notamment à l'écoute de leurs sentiments que l'on pourra citer le titre "Humain" qui nous incombe; Lamartine note des conditions:

"Avec des mors de fer ne brisez pas leurs bouches

سهام عبد الفتاح محمد جبر (Les sentiments religieux et mystiques Chez Alphonse de Lamartine) 
ne les écrasez pas sous de lourds fardeaux qu'ils vous lèchent la main et vous prêtent leur dos! adoucissez leurs mœurs en leur étant plus doux! ${ }^{86}$

\section{Effet du mysticisme religieux sur les animaux}

Cet appel aux sentiments stagnants dans les refends des cœurs ne connaît point de bornes. L'amour de Lamartine saute toutes les murailles; mais était-il prétentieux dans ses perspectives morales et sentimentales? Les points d'attache avec tous les êtres ne peuvent point nous laisser dans le doute:

"Nul de sait à moins d'avoir été bouvier, pasteur, soldat, chasseur ou solitaire comme moi, combien il y a d'amitié entre les animaux et leur maître. Ce monde est un océan de sympathies dont nous ne buvons qu'une goutte, quand nous pourrions en absorber des torrents. Depuis le cheval et le chien jusqu'à l'oiseau et depuis l'oiseau jusqu'à l'insecte, nous négligeons des milliers d'amis. Vous savez que moi je ne néglige pas ces amitiés, et que de la loge du dogue de basse-cour a l'étable du chevrier et de L'étable au mur du jardin ou je m'assied au soleil, connu des souris d'espalier, des belettes au museau flaireur, des reinettes a la voix d'argent, des clochettes du troupeau souterrain et des lézards, ces curieux aux fenêtres qui sortent la tête de toutes les fentes, j'ai des relations et des sentiments partout. Honni soit qui mal y pense. Je suis comme le vicaire de Goldsmith j'aime à aimer." 87

La place privilégiée du chien chez Lamartine, nous laisse aisément découvrir la finesse de cette relation. Le poète va trop loin dans l'appréciation de ces êtres car sa sensibilité à leur égard est illimitée. Nous découvrirons cette sensibilité dans sa reconnaissance à la clémence divine qui a fait de ces êtres inférieurs ,une source de consolation et d'amour:

سهام عبد الفتاح محمد جبر (Les sentiments religieux et mystiques Chez Alphonse de Lamartine) 
"Et par quelle pitié pour nos cœurs il te donne pour aimer encore ceux que n'aime plus personne". ${ }^{88}$

Le poète s'incline toujours devant le pouvoir du créateur. Dieu ne nous a pas préfères pour les humilier, les bafouer mais pour examiner jusqu'on peut aller notre savoir sur elles? Sommesnous dignes de notre prééminence? réside le dilemme. Lamartine est bien conscient de cette épreuve, l'éveil de son esprit sera sans répit:

"Aussi, pauvre animal, quoique a terre couche jamais d'un sot dédain mon pied ne t'a touche jamais d'un mot brutal contristant te tendresse Mon cour n'a repoussé ta touchante caresse." . ${ }^{89}$

Lamartine comprend parfaitement que s'il a été favorise à tant qu'être supérieur, il n'a pas le droit de sous-estimer aux qui n'ont pas eu cette chance innée. Ne sommes-nous pas tout à fait identiques à ces êtres inférieurs sur le plan physiologique? Rien ne nous sépare d'eux que la faculté de la raison Lamartine souligne consciencieusement cette similitude sur L'échelle de l'être:
"Mais, toujours, Ah! toujours en toi j'ai respecte de ton maître et du mien l'ineffable bonté comme on doit respecter sa moindre créature fière a quelque degré qu'ait voulu la nature. ${ }^{90}$

Grande leçon d'une fidélité hors pair, que celle donnée par le chien. Exemple admirable d'un sentiment qui devient rarissime de nos jours! Celui qui a créé les êtres est capable de préserver les bases morales de leur survie: 
"Dieu n'éteindra pas plus sa divine étincelle dans l'étoile des nuits dont la splendeur ruisselle que dans l'humble regard de ce tendre épagneul. ${ }^{91}$

Le pétillement des sentiments qui lient Lamartine au chien particulièrement ne cesse de nous éberluer. Le chien de Céder cherchant son maître partout, 1e trouva enfin dans la nuit. C'était la surprise, Céder le prend pour un lionceau et le tue. La fidélité de ce chien est décrite d'une façon plus qu'émouvante:

"Ainsi qu'il accourait sans avoir même bu

il était accouru, prompt à le reconnaître mourir pour son amour, de la main de son maître. ${ }^{92}$

La tragédie était extrêmement poignante pour Céder, interprète le "Code" moral Lamartinien. Il était plus que compatissant avec cet évènement:

"Pour le cour des mortels l'amour est un tel bien

qu'il ne peut sans saigner perdre celui d'un chien. ${ }^{93}$

Jocelyn de même n'aura plus que son chien à aimer:

"(...) Mets ton cour près du mien

et seuls à nous aimer aimons-nous pauvre chien." 94

Peu soucieux de surprendre et bien au contraire, spontané instinctif dans l'expression de ses sentiments; Lamartine est tout de même donner sa leçon à tous. Le public le moins instruit y compris.

Il n'hésitera pas alors à recourir aux évocations de l'imagerie populaire et versera un pleur au bout d'une tirade le "tendre épagneul":

"qui conduisait l'aveugle et meurt sur son cercueil"95

سهام عبد القناح محمد جبر (Les sentiments religieux et mystiques Chez Alphonse de Lamartine) 
Il n'aimait pas seulement les animaux mais aussi ceux les respectaient. De la provient son admiration pour les gens qui respectaient toutes les créatures. Les Védas étaient fort apprécies par Lamartine car ils trahissaient le respect de toute vie "inférieure" et font preuve d'une sincère sympathie à l'égard des animaux et des plantes:

"J'admirai, j'adorai cette parente universelle des êtres, cette fraternité de la vie entre tout ce qui sent, entre tout ce qui aime ici-bas dans la mesure de son intelligence et de sa destinée." 96

C'est ainsi, que grâce à ce mysticisme, Lamartine a découvert que la force de l'amour et de l'amitié est invincible, car elle est capable de surmonter les vagues du temps et du trépas, Quand les âmes s'unifient par l'Amour et l'Amitié, ils échappent à la notion du temps. Rien ne peut procurer cette transcendance de l'esprit que l'Amour et l'amitié dans l'existence terrestre. Cela était le souci majeur de Lamartine, il l'a exprimé par le truchement du souhait de Jocelyn:

"Ah! si je pouvais o Laurence

monter ou cette arche commence

gravir ces degrés éclatants

et pour qu'un ange m'y soutienne

1 'oeil au ciel, ma main dans la tienne

passer sur la mort et le temps !" 97

L'Amour est capable de nier la fin de la vie car son extase mystique peut nous dégager du magnétisme terrestre. Race à cette foi inflexible en Dieu et son Amour; Lamartine pulvérise le fil qui le séparait de sa fille disparue: 
"J'ai vu dans ses regards la flamme de la vie sous la main du trépas par dégréa assoupie se ramener encore au souffle de l'amour je disais chaque jour; Soleil, encore un jour !" 98

L'amour chez Lamartine c'est la survie de l'esprit; Il a bien expliqué cette conception à travers ses transports mystiques. Cette flamme divine ne peut jamais s'éteindre avec La mort, elle s'éclipse simplement avec la disparition de ceux qui s'aiment:

"L'homme n'est bien mort que quand tous ceux qui 1 'ont connu et aime sur la terre se sont couches, à leur tour dans le tombeau qui ne parle plus d'eux aux nouvelles générations." 99

Cette source d'énergie dans le cœur de Lamartine était tonnante d'Amour, tonitruante d'Amitié. Ecoutons le susurrement de son esprit, transperçant les voiles de la mort avec une sérénité ignorée des communs des mortels:

" J'étais un hymne vivant, criant, chantant,priant, invoquant, remerciant, adorant, débordaient en effusion, sans paroles, un cour ivre, une âme folle agitant, promenant au bord des abîmes un corps qui n'éprouvait plus sa matérialité, qui ne croyait plus, ni au temps, ni à l'espace, ni à la mort tant la vie de l'amour qui venait de jaillir en moi me donnait le sentiment ,la jouissance anticipée et la plénitude de l'immortalité ." 100

En quelques mots, on peut dire que Lamartine, était "un poète maître de son discours" ${ }^{\prime 101}$, un poète délicat, plus sensible que sensuel, grâce aux différents aspects du mysticisme 
religieux, déjà analysés, il exprime en beaux vers, des sentiments purs et pénétrant, inondant et forts .

Le mysticisme religieux fait de lui un poète chaste, sans effort et sans gaucherie puisqu' il le détail d'une distinction innée qui le menait, sans qu'il y songeât peut-être, à choisir parmi les émotions, les plus nobles et les plus sincères comme l'on vient de voir. 


\section{Marges}

1 --- Lamartine (Alphonse de): Les Confidences . Paris, Dondey Dupre ,1828 Voir T.IV , chapitre II PP. 74-75.

2 -- Lamartine : Jocelyn. Episode . Paris , éditions des Souscripteurs, 1848 . T..I

P.63

3 -- Lamartine (Alphonse de) : Les Confidences . op.cit Voir T.IV , chapitre VII ,P.49

4 -- Lamartine (Alphonse de) : Les Méditations poétiques, Paris, Editions des Souscripteurs, 1848 . P.256

5 - Ibid .p. 238.

${ }^{6}$-Lamartine : La Chute d'un Ange, Episode . Paris . Hachette, 1900 , P. 191.

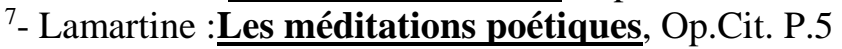

${ }^{8}$-Lamartine : Harmonies Poétiques et Religieuses , éditions des Souscripteurs 1848 ; T.II P. 25

9 - Ibid; p.30

10 -- Lamartine :Les méditations poétiques. Op.Cit. P.5

11 -Ibid. p.308

12 -Lamartine : La Chute d'un ange · op.cit .p.38

${ }^{13}$ Milton(J.) 1608 - 1674. Ce poète est auteur de : Poèmes philosophiques et pastoraux. Il a écrit un grand poème inspire de la Bible: Le Paradis Perdu

14 -Lamartine : La Chute d'un ange.Op.Cit. P.282

15- Lamartine:Cours familiers de litterature.Paris,Hachette,1912. P.220

16- Ibid; P.169

17- Ibid: P.319

18 _ Lamartine (A): Jocelyn. Episode. Op.cit.p. 123.

${ }^{19}$-Lamartine (A): Harmonies Poétiques et Religieuses. Op.citT.I,p.67,

${ }^{20}$ _Lamartine(A):Méditation Poétiques. Op.cit, p. 113.

21- Idem.

22_ Lamartine (A): Jocelyn. Episode. Op.cit, Tome I, P. 239.

23- Lamartine (A): Méditations Poétiques. Op.cit, p. 93.

24- Lamartine (A): Jocelyn. Episode. Op.cit, p. 157.

25_ Lamartine (A): Méditations Poétiques. Op.cit, p. 222.

26- Ibid p. 113.

27. Lamartine (A); Nouvelles Méditations Poétiques. Paris Editions des Souscripteurs. 1848, p. 113.

28 - Ibid. p. 35

${ }^{29}$-Ibid:p..319

30- Ibid .p.62

${ }^{31}$-Ibid:, p.319

سهام عبد الفتاح محمد جبر (Les sentiments religieux et mystiques Chez Alphonse de Lamartine) 
32_ Lamartine (A); Harmonies Poétiques et Religieuses. Op.cit. T.I,p.129.

33- Lamartine (A) : Recueillements poétiques. Paris, Editions des Souscripteurs. 1848, p, 64

34- Idem.

35 - Lamartine (A): Méditation Poétiques, op.cit, p. 89,

36. Idem.

37 - Lamartine (A): Jocelyn. Episode, op.cit, p, 208

38- Lamartine (A): Méditations Poétiques. Op.cit, p. 318

39. Lamartine (A): Jocelyn. Episode, op.cit, p, 229

40_ Ibid, p, 241

41- Ibid: p, 136

42 - Idem.

${ }^{43}$ - Ibid, p. 17.

44- Ibid; P.123

45 - Ibid. p. 176.

46- Lamartine (A): Méditations Poétiques. Op.cit, p. 269.

47- Lamartine (A): Nouvelles Méditations Poétiques. Op.cit, p. 261.

48_ Lamartine (A): Harmonies Poétiques et Religieuses. Op.cit,T.I,p.61.

49 - Ibid .p.127.

50- Ibid. p. 190

51 - Lamartine (.A): Jocelyn. Episode. Op.cit. Tome I, P 138.

52-Lamartine(A):Correspondance,Paris.Editions Dondey Dupré. 1856, Tome II, P 15.

53 - Idem.

54-Lamartine (A): Jocelyn. Episode. Op.cit, Tome I, p. 145.

55 - Ibid. p. 336.

56_ Lamartine (A): Recueillements Poétiques. Op.cit., p. 45.

57- Ibid; p. 336

58_ Lamartine (A): Nouvelles Meditations Poetiques. Paris. Editions des Souscripteurs.1848, p.8

59- Lamartine (A): Jocelyn. Episode. Op.cit Tome II p. 385.

60. Idem.

61. Idem.

62 - Idem

63_ Lamartine (A): Recueillements Poétiques. Op.cit, p.200.

64- Ibid. p.260.

65 - Idem.

66. Idem.

67 - Lamartine (A): Jocelyn. Episode. Op.cit, Tome I, p. 91.

68_ Ibid. p. 113

69. Lamartine (A): La Chute d'un Ange. Op.cit p. 226.

70- Lamartine (A): Méditations Poétiques op.cit, p. 217.

سهام عبد الفناح محمد جبر (Les sentiments religieux et mystiques Chez Alphonse de Lamartine) 
71_ Lamartine (A); Jocelyn. Episode. Op.cit. Tome I, p. 73.

72- Lamartine (A): Recueillements Poétiques. Op.cit, p. 34.

73 - Lamartine (A): Correspondance. Op.cit, Tome III, p. 18.

74- Lamartine (A): Recueillements Poétiques. Op.cit, p. 262.

75 - Ibid. p. 261.

76- Idem

77- Lamartine (A): La Chute d'un Ange. Op.cit, p. 261.

78 - Ibid. p. 225.

79 - Ibid: ,p. 50.

80_ Ibid;. p. 220.

81 - Idem.

82- Ibid .p. 131

83 - Ibid, p. 225

84- Ibid : p. 275.

85 - Ibid, p. 225

86_ Ibid..pp. 225-226.

87 - Lamartine (-A): Harmonies Poétiques et Religieuses. Op.cit. T.I, p,10.

88 - Lamartine (A): Jocelyn. Episode. Op.cit, Tome I, p. 109.

89. Idem.

90 - Idem.

91 - Idem.

92_ Lamartine(A): La Chute d'un Ange.op.cit,p. 159.

93. Idem.

94_ Lamartine (A); Jocelyn. Episode. Op.cit, Tome II, p. 16.

95- Ibid. p. 28

96- Lamartine (A): Cours familiers de littérature. Paris. Hachette, 1912. Tome III, p. 13.

97- Lamartine (A): Jocclyn Épisode. Op.cit, Tome I, p. 29.

98 - Lamartine (A): Méditations Poétiques. Op.cit, p. 35.

99- Lamartine (A): Nouvelles Méditations Poétiques. Op.cit, p. 8.

- Lamartine (A): Raphaël éd. Aurélie LOISELEUR, Paris, Gallimard, «Folio classique», 100 2011.P.14

- Ibid. p. $20^{101}$

سهام عبد الفتاح محمد جبر (Les sentiments religieux et mystiques Chez Alphonse de Lamartine) 


\section{Bibliographie}

\section{Corpus}

Lamartine (Alphonse de) :

Les Méditations poétiques __Paris , Editions des Souscripteurs, 1848 .

Harmonies poétiques et religieuses .Paris, Editions des Souscripteurs , 1848 .

Nouvelles, Méditations poétiques.Paris, Editions des Souscripteurs, 1848.

Recueillements poétiques.Paris, Editions des Souscripteurs , 1848 .

La Chute d'un Ang.Paris, Hachette, 1900.

Jocelyn. Episode Paris, Editions des Souscripteurs, 1848 .

Correspondances. Paris, Editions Dondey Dupré. 1856. Voir t. II.

Les Confidences. Paris, Dondey Dupré, 1828. Voir T.IV.

\section{Ouvrages consacrés à Lamartine}

سهام عبد الفتاح محمد جبر (Les sentiments religieux et mystiques Chez Alphonse de Lamartine) 
1- Boeninger (Yvonne) : Lamartine et le sentiment de la nature. Paris, Nizet et Bastard, 1954, 1 vol

2- Bouchard (Marcel) : Lamartine ou le sens de l'amour.

Paris, Les Belles Lettres, 1940, 1 vol

3- Dellanoy (Jean) : La Chute d'un Ange de Lamartine Thèse,Paris, 1956, 1 vol

4- Frejabile (Gustave ) : Les Meditations de Lamartine.

Paris, Malfére, 1931, 1 vol,

5- Grillet (Claudius.) : La Bible dans Lamartine.

Paris, e.vitte, 1938, 1 vol

6- Guyard ( Marius ) : Alphonse de Lamartine. Paris, éditions

Universitaires, 1956, 1 vol

7- Lucas ( Dubreton) : Lamartine. Paris, Flammarion 1955,1 vol

8- Maréchal (Christian) : Lamennais et Lamartine. Paris, 1907, 1 vol.

9- Lamartine: Raphaël. éd. Aurélie Loiseleur, Paris, Gallimard, «Folio classique», 2011,

\section{Sitographie:}

Alphonse de LAMARTINE - Comptoir Littéraire www.comptoirlitteraire.com > docs > 465-lamartine

Histoire des Girondins par Alp. de Lamartine

سهام عبد الفتاح محمد جبر (Les sentiments religieux et mystiques Chez Alphonse de Lamartine) 
books.google.com.eg > books

Alphonse de Lamartine, Méditations poétiques : Des ... www.lalanguefrancaise.com > lamartine > des-destinees-de-lapoesie

Alphonse de Lamartine, maître du romantisme - Watani en.wataninet.com, archive-articles , alphonse-de-lamartinemaitre-du...

Lamartine - Français - Fiches de Cours pour Lycée ... keepschool.com > Fiches de cours > Lycee > Francais

Alphonse de Lamartine - Docsity

www.docsity.com > ... > Appunti Liceo Linguistico > Appunti Francese

Oeuvres de Lamartine de l'Académie française books.google.com.eg > books 